\title{
A Survey of Psychiatrist's perception regarding the concept of 'Mind' and its position in psychiatry today
}

\author{
Shrirang Bakhle \\ ${ }^{1}$ Consultant Psychiatrist - Private Practice and Independent Researcher, Mumbai. \\ E-mail-ss.bakhle@gmail.com
}

\begin{abstract}
The term 'mind' is important in Psychiatry. For example, when certifying Testamentary Capacity, Psychiatrists certify that a person has a sound 'mind'. 'Mental' means 'related to mind'. With advances in neurobiology, Mental illness is being considered disease of brain. So are 'Mental' disorders, disorders of mind or brain? The study aims to understand Psychiatrists' perceptions about these fundamental issues: Is MSE done to know status of mind or brain? What is the difference between Psychiatry and Neurology if both study brain? Is 'mind' the difference? If Psychiatry is 'study of disorders of mind', then what is mind? What are the parts of mind? Is there any currently accepted framework or model of the mind? A questionnaire to assess the perceptions of Psychiatrists about these issues was presented to qualified Psychiatrists and Psychiatry residents. The replies were analysed to understand opinion trends and to see if there was consensus about these topics. There is clear consensus that Psychiatry and 'mental' disorders are concerned with 'mind'. However, there is complete lack of consensus about nature of mind and related topics. There is consensus that 'mental' disorders are not disorders of just brain. 'Mind' is important in Psychiatry. However, considering the complete lack of consensus about the nature of mind and related topics, there is an urgent need to debate and evolve consensus about these vital topics.
\end{abstract}

Key words: mind, psychiatry, brain, psychiatrists, perceptions, mind and brain.

\section{INTRODUCTION}

The term 'mind' is used often in Psychiatry. For example, when certifying Testamentary Capacity, the Psychiatrists have to say that a person has a sound 'mind' [1]. Historically, too, the science of Psychiatry evolved around the topic of mind. The National Institute of Health's (NIH) US National Library of Medicine Website describes the "Diseases of the Mind: Highlights of American Psychiatry through 1900". It says: "Benjamin Rush, often called "The father of American Psychiatry", wrote the first systematic textbook on mental diseases in America entitled, Medical Inquiries and Observations upon Diseases of the mind published in Philadelphia in 1812." Please note how the US NLM's webpage that describes the history of American Psychiatry is titled "Diseases of the Mind". And the Father of American Psychiatry wrote the first textbook of mental diseases describing the diseases of the mind [2].

However, in the last few decades, the focus in Psychiatry seems to have shifted to neurobiology and brain. The word 'mental', too, is used often in Psychiatry: Mental disorders, Mental Status Examination, Mental Health Act, National 
Institute of Mental Health, DSM of Mental Disorders (DSM 5) etc. The word 'mental', according to the Oxford Dictionary, means 'related to mind; related to disorders or illnesses of the mind' [3]. But banners put up at a recent Psychiatry conference said, "Mental illness is disease of the brain" [4]. This represents the view of the 'Biological Psychiatry'. So can we say that 'mental' disorders are disorders of mind or brain or both? Has 'mind' become redundant? Can Psychiatry replace the terms 'mind' and 'mental' with 'brain' and 'neurological'? If the 'Mental' disorders are disorders of the brain, then what is the difference between Neurology and Psychiatry? Why are some disorders of the brain (such as hemiplegia) not considered as 'mental' disorders?

The DSM-IV-TR, too, is not sure about this issue. The DSM is a manual of "mental' disorders. But it says, "...unfortunately the term (mental) persists in the title of DSM-IV-TR because we have not found an appropriate substitute" [5]. Thus the DSM-IV-TR wanted to remove the word 'mental' from its title but it could not find a more appropriate word. Brog and Guskin have reported a survey about "Medical students' judgments of mind and brain in the etiology and treatment of psychiatric disorders" [6]. However the study was about the perceptions of general medical students and not just members of the Psychiatric fraternity. There has been a study done on psychiatrists' concepts of mental illness but the focus of the study was the perceptions about the different aetiological models such as biological model, cognitive model, behavioural model etc [7]. Miresco and Kirmayer surveyed Psychiatrists' and Psychologists' opinions about the Mind - Brain dichotomy in the aetiology of mental illness using clinical vignettes. Their analysis was also related to whether they tend to put the blame on the patients depending on whether the aetiology was perceived as primarily psychological or neurobiological [8].

A survey of literature and discussions with eminent Psychiatrists from India and abroad, revealed different opinions about the topic of 'mind'. Hence the present study was planned to crystallize the viewpoints. The present study aims to understand the perceptions of Psychiatrists about these fundamental issues in Psychiatry: Is the topic of 'mind' still relevant in today's Psychiatry? If so, then what is mind? What are the relationships between mind-brain and mind-behaviour? What is the best method of studying the mind? If Psychiatry is to be considered as 'the study of diseases of the brain', then what is the difference between Psychiatry and Neurology? Is mind the difference?

\section{METHODOLOGY}

A questionnaire was prepared to understand the perceptions of Psychiatrists about these topics. It had a total of 15 questions. The first group of 4 questions aims to understand the perceptions about the similarities and differences between Biological Psychiatry (with its focus on the brain) and Neurology which is also the study of brain. The next 2 questions are about understanding the perceptions about the word 'mental' - as in 'Mental' disorders and the Mental Status Examination. The next 7 questions probe the perceptions about the nature of mind, its relationship with brain and behaviour and the best method of studying the mind of the patient. The last 2 questions deal with the 'Mental' disorders and brain. The preliminary discussions had revealed a variety of opinions, and since the aim of the study is to understand the perceptions about these topics, the questions were deliberately kept open-ended. Options were offered in only two of the questions and they, too, have an option of 'any other'. The participants in Psychiatry conferences form the background population. They were offered the questionnaire. The respondents voluntarily filled up the questionnaire. 
The response rate was $60 \%$. The form briefly introduced the purpose of the study. The respondents were assured about confidentiality. The results have been described using simple and basic descriptive statistics.

\section{RESULTS}

Over $75 \%$ of the psychiatrists in the survey were below the age of 40 years. Thus most opinions were from younger psychiatrists. Majority were male. A total of 36 psychiatrists were surveyed in this pilot study. Mean age of the psychiatrists surveyed was 34.8 years. Since most of the questions were open ended the answers were analyzed for similarities while differences were duly recognized as well. The main findings of the survey are highlighted in table 1 . Over $75 \%$ felt that psychiatry involved disorders of the mind and no one gave brain alone as a sole answer though many mentioned both mind and brain. $80.5 \%$ felt that the word mental correlated with the mind. $88.8 \%$ agreed in the existence of an entity called mind. 33 out of 36 psychiatrists gave different answers when asked what they meant by mind. 3 did not answer the question. When asked about what were the parts of the mind, 26 out of 36 gave different answers while 10 did not answer. $66 \%$ admitted that there was no unified theory of the mind accepted by psychiatrists and all had diverse views. $50 \%$ also felt that psychology too was a study of the mind and/or psyche. It was interesting to note that when asked about the differences between psychiatric and neurological disorders, $30.5 \%$ felt that psychiatric disorders lacked a structural certainty and correlation in the brain. $75 \%$ believed that there were no neuroimaging modalities that would help us image the mind and all we could see was the brain. When asked on the relationship between the mind and the brain diverse answers were received. When asked whether diagnostic criteria of psychiatry must be based on symptoms, brain dysfunction or mind dysfunction, there was a divided view.

\section{DISCUSSION}

There is consensus that Psychiatry and 'mental' disorders are concerned with 'mind'. However, there is a complete lack of consensus about the nature of mind and related topics. On the other hand, there is complete consensus that 'mental' disorders are not disorders of just brain. There is also consensus that the diagnostic criteria of 'mental' disorders cannot be defined in terms of brain markers. It is quite illuminating to study the spectrum of perceptions about these vital topics. This is a pilot study with a small sample size. Hence the conclusions need to be confirmed with larger studies. However, the clear cut trends - both the consensus and the lack of it - are worth studying. A better representation from across the country and abroad will make a future study more representative. The term 'mind' is used often in Psychiatry. For example, when certifying Testamentary Capacity, the Psychiatrists have to say that a person has a sound 'mind'. Considering the complete lack of consensus today about 'mind' and related topics, there is an urgent need to debate and evolve consensus about this vital topic in Psychiatry. 


\section{Table 1 - Summary of the main findings}

\section{Main findings of the survey on 'Mind'}

- $75 \%$ felt that psychiatry was concerned with the mind. No one gave brain as a sole answer.

- $80.5 \%$ felt that mental denoted the mind.

- $88.8 \%$ believed firmly in an entity called the mind.

- $66.6 \%$ opined that there is no universal theory of the mind.

- $75 \%$ felt that brain dysfunction alone cannot elucidate psychiatric disorders

\section{REFERENCES}

1. Jiloha RC. Mental capacity/testamentary capacity. Clinical Practice Guidelines of the Indian Psychiatric Society.

2. Ozarin L. North M. (Eds.) Diseases of the mind: Highlights of American Psychiatry through 1900. Viewed on the US National Library of Medicine website.

3. The Oxford English Dictionary. Oxford: Oxford University Press; 2010.

4. De Sousa A. Towards an integrative theory of consciousness Part 1 : neurobiological and cognitive models. Mens Sana Monogr 2013;11:100-50.

5. American Psychiatric Association. Diagnostic and Statistical Manual of Mental Disorders Fourth Edition Text Revised. American Psychiatric Publishing; 2000.

6. Brog MA, Guskin KA. Medical Students' Judgments of Mind and Brain in the etiology and Treatment of Psychiatric Disorders: A Pilot Study. Acad Psychiatry 1998;22:229-35.

7. Harland R. A study of psychiatrists' concepts of mental illness. Psychol Med 2009;39:96776.

8. Miresco MI, Kirmayer LI. The persistence of mind-brain dualism in Psychiatric reasoning about clinical scenarios. Am J Psychiatry 2006;163:913-8..

Acknowledgements - Nil

Conflict of Interest - Nil

Funding - Nil. 